\title{
DYNAMIC PRESSURES ON ACCELERATED FLUID CONTAINERS
}

By G. W. HousNer

\begin{abstract}
An analysis is presented of the hydrodynamic pressures developed when a fluid container is subjected to horizontal accelerations. Simplified formulas are given for containers having twofold symmetry, for dams with sloping faces, and for flexible retaining walls. The analysis includes both impulsive and convective fluid pressures.
\end{abstract}

\section{INTRODUCTION}

The DYNAMIC fluid pressures developed during an earthquake are of importance in the design of structures such as dams and tanks. The first solution of such a problem was that by Westergaard (1933), who determined the pressures on a rectangular, vertical dam subjected to horizontal acceleration. Jacobsen (1949) solved the corresponding problem for a cylindrical tank containing fluid and for a cylindrical pier surrounded by fluid. Werner and Sundquist (1949) extended Jacobsen's work to include a rectangular container, a semicircular trough, a triangular trough, and a hemisphere. Graham and Rodriguez (1952) gave a very thorough analysis of the impulsive and convective pressures in a rectangular container. Hoskins and Jacobsen (1934) determined impulsive fluid pressures experimentally, and Jacobsen and Ayre (1951) gave the results of similar measurements. Zangar (1953) presented the pressures on dam faces as measured on an electric analog.

The foregoing analyses were all carried out in the same fashion, which requires finding a solution of La Place's equation that satisfies the boundary conditions. With these known solutions as checks on accuracy, it is possible to derive satisfactory solutions by an approximate method which avoids partial-differential equations and infinite series and presents solutions in simple forms. The approximate method appeals to physical intuition and makes it easy to visualize the fluid motion, and it thus seems particularly suitable for engineering applications. To introduce the method, the problem of the rectangular tank is treated in some detail; applications to other types of containers are treated more concisely.

The more exact analyses show that the pressures can be separated into impulsive and convective parts. The impulsive pressures are those associated with the forces of inertia produced by impulsive movements of the walls of the container, and the pressures developed are directly proportional to the acceleration of the container walls. The convective pressures are those produced by the oscillation of the fluid and are thus the consequences of the impulsive pressures. In the following analysis the impulsive and convective pressures are examined separately, the fluid is assumed to be incompressible, and fluid displacements are assumed to be small.

\section{Impulsive Pressures}

Consider a container with vertical side walls and horizontal bottom that is symmetrical with respect to the vertical $x-y$ and $z-y$ planes. Let the walls of the container be given an impulsive acceleration $\dot{u}_{0}$ in the $\mathrm{x}$ direction. This will generate

\footnotetext{
Manuscript received for publication November 17, 1955.
} 
fluid acceleration $\dot{u}, \dot{v}$ in the $\mathrm{x}, \mathrm{y}$ directions and may also generate an acceleration component $\dot{w}$ in the $\mathrm{z}$ direction. For a rectangular tank $\dot{w}$ is obviously zero, and Jacobsen (1949) showed that for a cylindrical tank $\dot{w}$ is also zero. In what follows it will be assumed that the ratio of $\dot{w}$ to $\dot{u}$ is either exactly zero or at least so small that $\dot{w}$ may be neglected. Physically, this is equivalent to having the fluid restrained by thin, vertical membranes, spaced $d z$ apart, which force the fluid motion to take

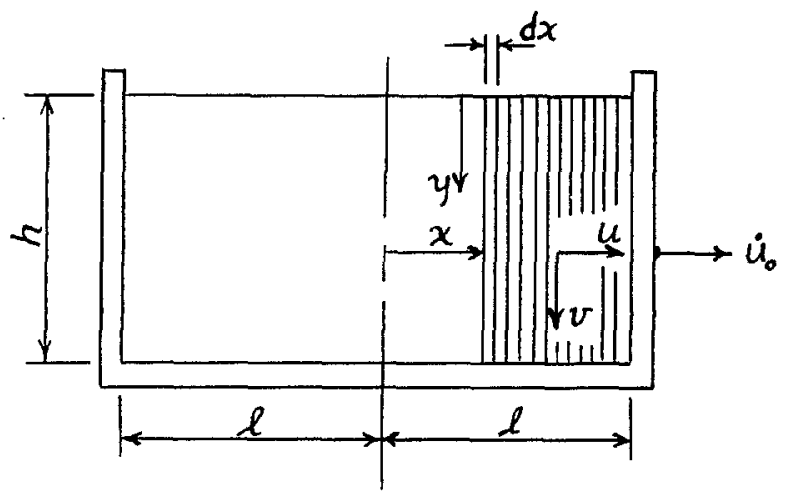

Fig. 1.

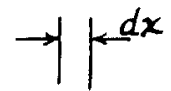

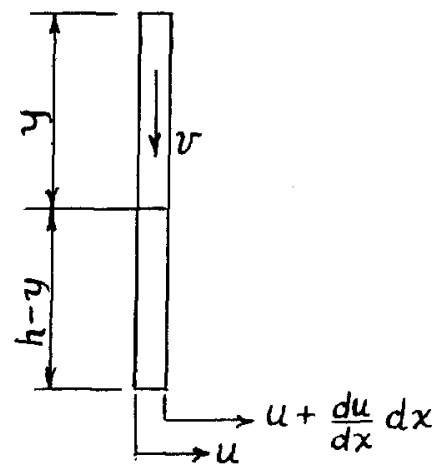

Fig. 2.

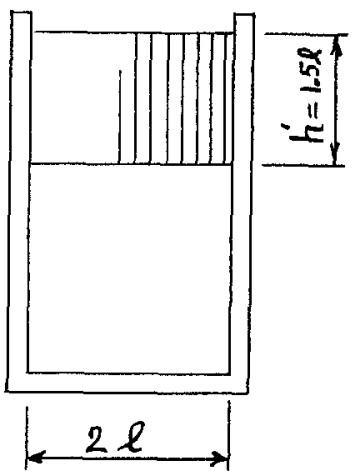

Fig. 3 .

place in the $x, y$ plane only. It is then sufficient to consider the impulsive pressures generated in a lamina of fluid.

Consider a lamina of fluid of unit thickness, figure 1, and let the walls be given a horizontal acceleration $\dot{u}_{0}$. The initial effect of this acceleration is to impart a horizontal acceleration to the fluid and also a vertical component of acceleration. This action of the fluid is similar to that which would result if the horizontal component, $u$, of fluid velocity were independent of the $y$ coördinate; that is, imagine the fluid to be constrained by thin, massless, vertical membranes free to move in the $\mathrm{x}$ direction, and let the membranes be originally spaced a distance $d x$ apart. When the walls of the container are given an acceleration, the membranes will be accelerated with the fluid, and fluid will also be squeezed vertically with respect to the membranes. As shown in figure 2, the fluid constrained between two adjacent mem- 
branes is given a vertical velocity

$$
v=(h-y) \frac{d u}{d x}
$$

Since the fluid is incompressible, the accelerations satisfy the same equation, so

$$
\dot{v}=(h-y) \frac{d \dot{u}}{d x}
$$

The pressure in the fluid is then given by

$$
\frac{\partial p}{\partial y}=-\rho \dot{v}
$$

where $\rho$ is the density of the fluid. The total horizontal force on one membrane is

$$
P=\int_{0}^{h} p d y
$$

These equations may be written

$$
\begin{aligned}
& \dot{v}=(h-y) \frac{d \dot{u}}{d x} \\
& p=-\rho \int_{0}^{y}(h-y) \frac{d \dot{u}}{d x} d y=-\rho h^{2}\left(y / h-\frac{1}{2}(y / h)^{2}\right) \frac{d \dot{u}}{d x} \\
& P=-\rho h^{2} \int_{0}^{h}\left(y / h-\frac{1}{2}(y / h)^{2}\right) \frac{d \dot{u}}{d x} d y=-\rho h^{3} / 3 \frac{d \dot{u}}{d x}
\end{aligned}
$$

The acceleration $\dot{u}$ is determined from the horizontal motion of the fluid contained between two membranes. The slice of fluid shown in figure 2 will be accelerated in the $\mathrm{x}$ direction if the pressures on the two faces differ. The equation of motion is

$$
\frac{d P}{d x} d x=-\rho h d x \dot{u}
$$

Using the value of $P$ from equation (4) gives

$$
\frac{d^{2} \dot{u}}{d x^{2}}-\frac{3}{h^{2}} \dot{u}=0
$$

and the solution of this equation is

$$
\dot{u}=C_{1} \cosh \sqrt{3} \frac{x}{h}+C_{2} \sinh \sqrt{3} \frac{x}{h}
$$

Equations (4) and (6) determine the fluid pressures, and they are strictly applicable only when the surface of the fluid is horizontäl, but if consideration is restricted to small displacements of fluid the equations may be used even when the surface of the fluid has been excited into motion, that is, equations (4) give the impulsive flujd pressures, $p(t)$, corresponding to arbitrary acceleration $\dot{u}_{0}(t)$.

If the container is slender, having $h>1.5 l$, somewhat better results are obtained 


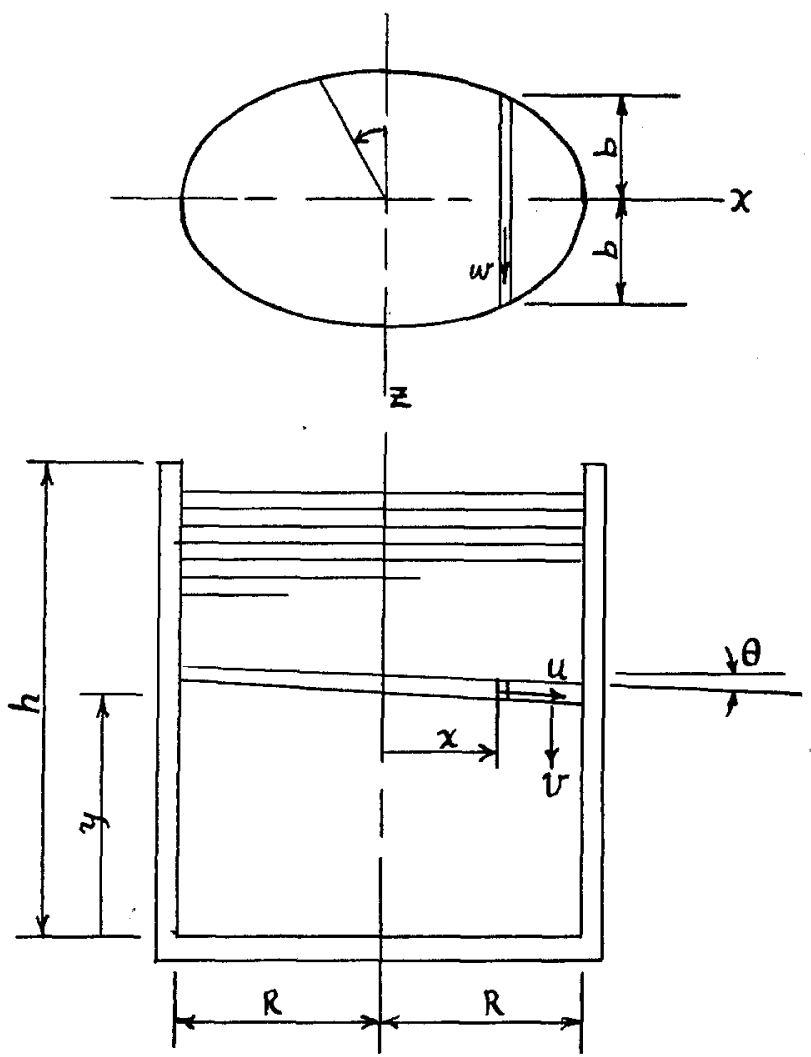

Fig. 4.

by applying equations (4) to the upper portion, $h^{\prime}=1.5 l$, of the fluid only and considering the fluid below this point to move as a completely constrained fluid exerting a wall pressure $p_{w}=\rho l \dot{u}_{0}$ (see fig. 3 ). At a depth of $1.5 l$ the moment exerted on the horizontal plane by the fluid above is approximately equal to the moment $\left(\frac{2}{3} \rho \dot{u}_{0} l^{3}\right)$ exerted on the same plane by the constrained fluid below which implies that the generation of fluid velocity is restricted essentially to the fluid in the upper part of a slender container.

\section{Convective Pressures}

When the walls of a fluid container are subjected to accelerations, the fluid itself is excited into oscillations and this motion produces pressures on the walls and floor of the container. To examine the first mode of vibration of the fluid consider constraints to be provided by horizontal, rigid membranes, free to rotate, as shown in figure 4. Let $u, v, w$ be the $\mathrm{x}, \mathrm{y}, \mathrm{z}$ components of fluid velocity, and describe the constraints on the flow by the following equations:

$$
\begin{gathered}
\frac{\partial(u b)}{\partial x}=-b \frac{\partial v}{\partial y} \\
v=x \dot{\theta} \\
\frac{\partial w}{\partial z}=-\left(\frac{\partial u}{\partial x}+\frac{\partial v}{\partial y}\right)
\end{gathered}
$$


where $b$ and $\theta$ are as shown in figure 4. These equations state, respectively, that the fluid at a given $x, y$ moves with a uniform $u$, that all the fluid at a given $x, y$ moves with the same $v$, and that continuity of flow is preserved. In a manner similar to that of the preceding section the appropriate equations of motion could be written for the particular shape of container under consideration. A general solution, applicable to any shape (twofold symmetry) can be deduced as follows. From the preceding equations

$$
\begin{aligned}
& u=-\frac{1}{b} \frac{\partial \dot{\theta}}{\partial y} \int_{-R}^{x} x b d x \\
& w=z \frac{b^{\prime}}{b^{2}} \frac{\partial \dot{\theta}}{\partial y} \int_{-R}^{x} x b d x
\end{aligned}
$$

where $b^{\prime}=d b / d x$. The total kinetic energy is thus:

$$
\begin{aligned}
T & =\frac{1}{2} \rho \int_{0}^{h} \int_{-R}^{+R} \int_{-b}^{+b}\left\{x^{2} \dot{\theta}^{2}+\frac{1}{b^{2}}\left(\frac{\partial \dot{\theta}}{\partial y}\right)^{2}\left(\int_{-R}^{x} x b d x\right)^{2}\left(1+z^{2}\left(\frac{b^{\prime}}{b}\right)^{2}\right)\right\} d x d y d z \\
& =\frac{1}{2} \rho \int_{0}^{h}\left\{I_{z} \dot{\theta}^{2}+K\left(\frac{\partial \dot{\theta}}{\partial y}\right)^{2}\right\} d y
\end{aligned}
$$

where

$$
\begin{aligned}
& I_{z}=\int_{A} x^{2} d A \\
& K=2 \int_{-R}^{+R} \frac{1}{b}\left(\int_{-R}^{x} b x d x\right)^{2}\left(1+\frac{b^{\prime 2}}{3}\right) d x
\end{aligned}
$$

The potential energy of the fluid is

By Hamilton's Principle

$$
V=\frac{1}{2} \rho g \theta_{h}^{2} \int x^{2} d x d z=\frac{1}{2} \rho g \theta_{h}^{2} I_{z}
$$

$$
\begin{aligned}
& \delta \int_{t_{1}}^{t_{2}}(T-V) d t=0 \\
& \delta \int_{t_{1}}^{t_{2}} \frac{1}{2} \rho\left\{\int_{0}^{h}\left[I_{z} \dot{\theta}^{2}+\left(\frac{\partial \dot{\theta}}{\partial y}\right)^{2} K\right] d y-g \theta_{h}^{2} I_{z}\right\} d t=0
\end{aligned}
$$

or

$$
\int_{t_{1}}^{t_{2}} \int_{0}^{h} \rho\left(I_{z} \dot{\theta}-K \frac{\partial^{2} \dot{\theta}}{\partial y^{2}}\right) \delta \dot{\theta} d x d t+\int_{t_{1}}^{t_{2}} \rho\left(K\left(\frac{\partial \ddot{\theta}}{\partial y}\right)_{h}+g I_{z} \theta_{h}\right) \delta \theta_{h} d t=0
$$

This gives the two equations

$$
\begin{aligned}
& \frac{\partial^{2} \dot{\theta}}{\partial y^{2}}-\frac{I_{z}}{K} \dot{\theta}=0 \\
& \frac{\partial^{2}}{\partial t^{2}}\left(\frac{\partial \theta}{\partial y}\right)_{h}+g \frac{I_{z}}{K} \theta_{h}=0
\end{aligned}
$$


From which there is obtained by integration

$$
\begin{gathered}
\theta=\theta_{h} \frac{\sinh \sqrt{\frac{I_{z}}{K}} y}{\sinh \sqrt{\frac{I_{z}}{K}} h} \sin \omega t \\
\omega^{2}=g \sqrt{\frac{I_{z}}{K}} \tanh \sqrt{\frac{I_{z}}{K}} h
\end{gathered}
$$

These are the equations for the free oscillation and the natural frequency of the fundamental mode of vibration. For a container of specified shape, such as rectangular, circular, elliptical, etc., it is necessary to evaluate only the integrals $I_{z}$ and $K$.

The pressure in the fluid is given by

$$
\begin{aligned}
\frac{\partial p}{\partial z} & =-\rho \dot{w} \quad \frac{\partial p}{\partial x}=-\rho \dot{u} \\
p & =-\rho \frac{\partial \ddot{\theta}}{\partial y}\left\{-\int \frac{Q}{b} d x+\frac{z^{2}}{2} \frac{b^{\prime}}{b^{2}} Q\right\} \\
Q & =\int_{-R}^{x} x b d x
\end{aligned}
$$

Knowing $p$, the forces and moments on the walls and floor of the container can be determined readily.

\section{Rectangular Container}

For a rectangular container of unit width as shown in figure 1, the boundary conditions for the impulsive pressures are $\dot{u}=\dot{u}_{0}$ at $x= \pm l$, for which equation (6) gives

Equations (4) then give

$$
\dot{u}=\dot{u}_{0} \frac{\cosh \sqrt{3} \frac{x}{h}}{\cosh \sqrt{3} \frac{l}{h}}
$$

$$
\begin{aligned}
& p=-\rho \dot{u}_{0} h \sqrt{3}\left(y / h-\frac{1}{2}(y / h)^{2}\right) \frac{\sinh \sqrt{3} \frac{x}{h}}{\cosh \sqrt{3} \frac{l}{h}} \\
& P=-\rho \dot{u}_{0} \frac{h^{2}}{3} \frac{\sinh \sqrt{3} \frac{x}{h}}{\cosh \sqrt{3} \frac{l}{h}}
\end{aligned}
$$

The wall acceleration, $\dot{u}_{0}$, thus produces an increase of pressure on one wall and a decrease of pressure on the opposite wall of 


$$
p_{w}=\rho i_{0} h\left(y / h-\frac{1}{2}(y / h)^{2}\right) \sqrt{3} \tanh \sqrt{3} \frac{l}{h}
$$

and produces a pressure on the bottom of the tank

$$
p_{b}=-\rho \dot{u}_{0} h \frac{\sqrt{3}}{2} \frac{\sinh \sqrt{3} \frac{x}{h}}{\cosh \sqrt{3} \frac{l}{h}}
$$

The total force acting on one wall is

$$
P=\rho \dot{u}_{0} \frac{h^{2}}{\sqrt{3}} \tanh \sqrt{3} \frac{l}{h}
$$

and its resultant acts at a distance above the bottom

$$
h_{0}=\frac{3}{8} h \quad\left(\frac{h}{l}<1.5\right)
$$

It is seen that the over-all effect of the fluid on the walls of the container is the same as if a fraction, $2 P \div 2 l h \rho \dot{u}_{0}$, of the total mass of the fluid were fastened rigidly to the walls of the container at a height $3 / 8 h$ above the bottom. The magnitude of this equivalent mass, $M_{0}$, is

$$
M_{0}=M \frac{\tanh \sqrt{3} \frac{l}{h}}{\sqrt{3} \frac{l}{h}}
$$

where $M$ is the total mass of the fluid.

The total moment exerted on the bottom of the tank is

$$
\int_{-l}^{+l} x p_{b} d x=-\rho \dot{u}_{0} h^{2} l\left(1-\frac{\tanh \sqrt{3} \frac{l}{h}}{\sqrt{3} \frac{l}{h}}\right)
$$

Including this, the correct total moment on the tank is given when the equivalent mass $M_{0}$ is at an elevation above the bottom of

$$
h_{0}=\frac{3}{8} h\left(1+\frac{4}{3}\left(\frac{\sqrt{3} \frac{l}{h}}{\tanh \sqrt{3} \frac{l}{h}}-1\right)\right)
$$

The accuracy of the preceding analysis can be judged by comparison with the values computed by Graham and Rodriguez (1952). Equation (18) gives an $M_{0}$ slightly larger than that computed by these authors with maximum error less than 2.5 per cent, and equation (20) gives an $h_{0}$ slightly smaller than theirs with a maximum error less than 2 per cent. It may thus be concluded that for the rectangular tank the errors introduced by the approximation of equation (1) are negligible so far as engineering purposes are concerned. 
In the case of free oscillations of the fluid in the fundamental mode for a rectangular tank of unit width, equations (9) are

$$
\begin{aligned}
& I_{z}=\int_{-l_{1}}^{+l} x^{2} d x=\frac{2}{3} l^{2} \\
& K=2 \int_{-l}^{+l} 2\left(\int_{-l}^{+l} \frac{x}{2} d x\right)^{2} d x=\frac{4}{15} l^{5}
\end{aligned}
$$

thus $\sqrt{\frac{I_{z}}{K}}=\sqrt{\frac{5}{2}} \frac{1}{l}$ and equations (10a) are

$$
\begin{aligned}
& \theta=\theta_{h} \frac{\sinh \sqrt{\frac{5}{2}} \frac{y}{l}}{\sinh \sqrt{\frac{5}{2}} \frac{h}{l}} \sin \omega t \\
& \omega^{2}=\sqrt{\frac{5}{2}} \frac{g}{l} \tanh \sqrt{\frac{5}{2}} \frac{h}{l}
\end{aligned}
$$

The velocity at any point in the fluid is given by

$$
\begin{aligned}
& u=\frac{l^{2}-x^{2}}{2} \frac{d \dot{\theta}}{d y} \\
& v=\dot{\theta} x
\end{aligned}
$$

The pressure in the fluid is given by

$$
\begin{aligned}
\frac{\partial p}{\partial x} & =-\rho \dot{u} \\
p & =-\rho \frac{l^{3}}{2}\left(\frac{x}{l}-\frac{1}{3}\left(\frac{x}{l}\right)^{3}\right) \frac{d \ddot{\theta}}{d y}
\end{aligned}
$$

The pressure exerted on the wall of the container, $(x=l)$, is

$$
p_{w}=\rho \frac{l^{2}}{3} \sqrt{\frac{5}{2}} \frac{\cosh \sqrt{\frac{5}{2}} \frac{y}{l}}{\sinh } \omega^{2} \theta_{h} \sin \omega t
$$

The force exerted on one wall is

$$
P=\int_{0}^{h} p_{w} d y=\rho \frac{l^{3}}{3} \omega^{2} \theta_{h} \sin \omega t
$$

The total force, $2 P$, exerted on the tank by the fluid is the same as would be produced by an equivalent mass $M_{1}$ that is spring mounted as shown in figure 5 . If $M_{1}$ 
oscillates with displacement $x_{1}$ the force against the tank and the kinetic energy of the mass are as follows:

$$
\begin{aligned}
& x_{1}=A_{1} \sin \omega t \\
& F_{1}=-M_{1} A_{1} \omega^{2} \sin \omega t \\
& T=\frac{1}{2} M_{1} A_{1}^{2} \omega^{2} \sin ^{2} \omega t
\end{aligned}
$$

Comparing these with the corresponding equations for the oscillating fluid it is seen that

$$
\begin{aligned}
& A_{1}=\theta_{h} \frac{h}{\sqrt{\frac{5}{2}} \frac{h}{l} \tanh \sqrt{\frac{5}{2}} \frac{h}{l}} \\
& M_{1}=M\left(\frac{1}{3} \sqrt{\frac{5}{2}} \frac{l}{h} \tanh \sqrt{\frac{5}{2}} \frac{h}{l}\right)
\end{aligned}
$$

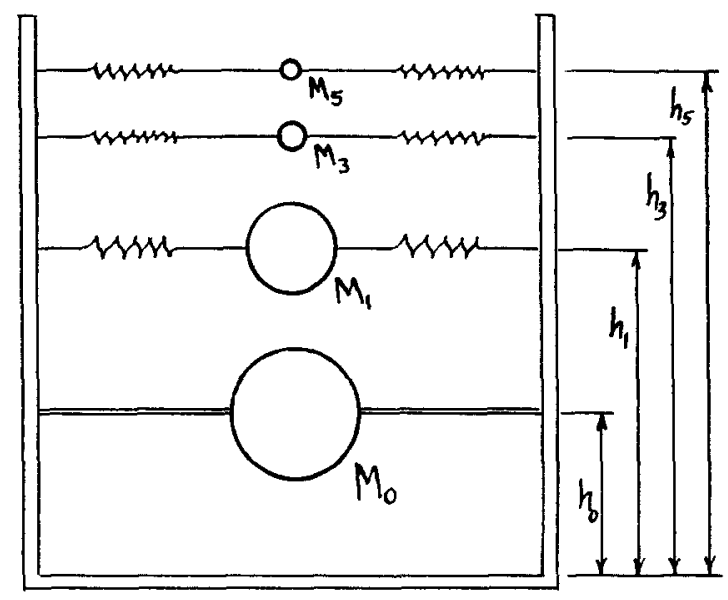

Fig. 5.

The elevation of $M_{1}$ above the bottom of the tank is determined so that it produces the same moment as the fluid. Considering only the moment of the fluid pressures on the walls (neglecting the pressures on the bottom), there is obtained

$$
h_{1}=h\left(1-\frac{1}{\sqrt{\frac{5}{2}} \frac{h}{l} \tanh \sqrt{\frac{5}{2}} \frac{h}{l}}\right)
$$

When the pressures exerted on the bottom are also taken into account the height is

$$
h_{1}=h\left(1-\frac{\cosh \sqrt{\frac{5}{2} \frac{h}{l}-2}}{\sqrt{\frac{5}{2} \frac{h}{l}} \sinh \sqrt{\frac{5}{2}} \frac{h}{l}}\right)
$$


Comparing with the exact solution of Graham and Rodriguez, it is found that equation (21) gives a value for $\omega^{2}$ that is slightly too large with a maximum error less than 1 per cent; equation (26) gives a value of $M_{1}$ slightly too large with a maximum error less than 2 per cent.

As shown in figure 5, the over-all effect of the fluid upon the container is the same as a system consisting of the container, a fixed mass $M_{0}$, and spring-mounted masses $M_{1}, M_{3}$; etc. It will be noted that the formulas for the higher unsymmetrical $(n=1,3,5 \cdots)$ modes are the same as for the first mode if $l$ is replaced by $l / n$.

The response of the system shown in Figure 5 when the container is subjected to arbitrary horizontal acceleration can be computed readily. From the motion of $M_{1}$, the oscillation of the fluid in the fundamental mode can be determined from equation (26), which gives the relation between $A_{1}$ and $\theta_{h}$. The actual displacement of the water surface is determined from equation (22), which at $y=h$ gives

$$
p_{h}=\rho \frac{l^{3}}{2}\left(x / l-\frac{1}{3}(x / l)^{3}\right) \omega^{2} \theta_{h} \sin \omega t
$$

This pressure is produced by the weight and inertia force of the fluid above the plane $y=h$. The depth $d$ of water above this plane is thus

$$
d=\frac{p_{h}}{\rho\left(g-x \ddot{\theta}_{h}\right)}
$$

\section{Cylindrical Container}

Consider a cylindrical tank as shown in figure 6 , subjected to a horizontal acceleration $\dot{u}_{0}$ and let the fluid be constrained between fixed membranes parallel to the $\mathrm{x}$ axis. Jacobsen (1949) has shown that an impulse $\dot{u}_{0}$ does not generate a velocity component $\dot{w}$ in the fluid so that in this case the membranes do not actually introduce a constraint. Fach slice of fluid may thus be treated as if it were a narrow rectangular tank and the equations of the preceding section will apply. The pressure exerted against the wall of the tank is, from equation (14),

$$
p_{w}=-\rho \dot{u_{0}} h\left(y / h-\frac{1}{2}(y / h)^{2}\right) \sqrt{3} \tanh \left(\sqrt{3} \frac{R}{h} \cos \phi\right)
$$

The pressure on the bottom of the tank is

$$
p_{b}=-\rho \dot{u}_{0} h \frac{\sqrt{3}}{2} \frac{\sinh \sqrt{3} \frac{x}{h}}{\cosh \sqrt{3} \frac{l}{h}}
$$

The preceding expressions are not convenient for calculating the total force exerted by the fluid. The following modification gives very accurate values for $R / h$ small and somewhat overestimates the pressure when $R / h$ is not small.

$$
p_{w}=-\rho \dot{u}_{0} h\left(y / h-\frac{1}{2}(y / h)^{2}\right) \sqrt{3} \cos \phi \tanh \sqrt{3} \frac{R}{h}
$$


From this expression the resultant force exerted on the wall is

$$
P=\int_{0}^{h} \int_{0}^{2 \pi} p_{w} \cos \phi R d \phi d y=-\rho \dot{u}_{0} \pi R^{2} h \frac{\tanh \sqrt{3} \frac{R}{h}}{\sqrt{3} \frac{R}{h}}
$$

from which it is seen that the force exerted is the same as if an equivalent mass $M_{0}$ were moving with the tank, where
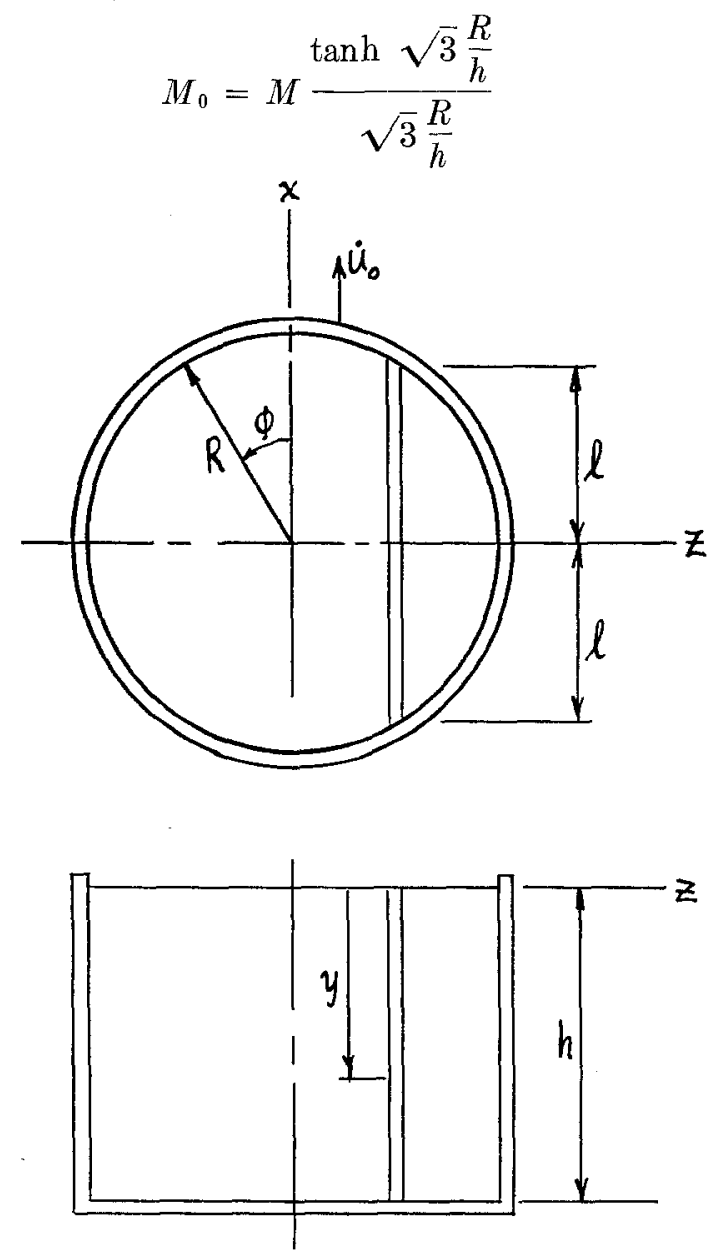

Fig. 6.

Comparing with Jacobsen (1949), it is found that equation (34) overestimates $M_{0}$ with a maximum error less than 4 per cent.

To exert a moment equal to that exerted by the fluid pressure on the wall, the mass $M_{0}$ should be at a height above the bottom

$$
h_{0}=\frac{3}{8} h \quad\left(\frac{h}{R}<1.5\right)
$$


If the moment exerted by the pressures on the tank bottom are included, the equivalent mass, $M_{0}$, must be at a height

$$
h_{0}=\frac{3}{8} h\left(1+\frac{4}{3}\left(\frac{\sqrt{3} \frac{R}{h}}{\tanh \sqrt{3} \frac{R}{h}}\right)-1\right) \quad\left(\frac{h}{R} \overline{<} 1.5\right)
$$

to produce the proper total moment on the tank. Comparing with Jacobsen (1949) it is found that equation (36) underestimates $h_{0}$ with a maximum error less than 6 per cent.

The free oscillations of the fluid (first mode) are determined from equations (21), etc. For the cylindrical tank

$$
\begin{aligned}
I_{z} & =\frac{\pi R^{4}}{4} \quad K=\frac{2 \pi}{27} R^{6} \quad \sqrt{\frac{I_{z}}{K}}=\sqrt{\frac{27}{8}} \frac{h}{R} \\
\omega^{2} & =\frac{g}{R} \sqrt{\frac{27}{8}} \tanh \sqrt{\frac{27}{8}} \frac{h}{R} \\
\theta & =\theta_{h} \frac{\sinh \sqrt{\frac{27}{8}} \frac{y}{R}}{\sinh \sqrt{\frac{27}{8}} \frac{h}{R}}
\end{aligned}
$$

Comparing with the exact solution, Lamb (1932), it is found that equation (37) slightly overestimates $\omega^{2}$ with a maximum error less than 1 per cent.

From equations (11) the pressure in the fluid is given by

$$
\begin{gathered}
p=-\rho \frac{R^{3}}{3}\left(\frac{x}{R}-\frac{1}{3}\left(\frac{x}{R}\right)^{3}-\frac{1}{4} \frac{x}{R}\left(\frac{z}{R}\right)^{2}\right) \frac{\partial \ddot{\theta}}{\partial y} \\
\frac{\partial \ddot{\theta}}{\partial y}=-\left(\sqrt{\frac{27}{8}} \frac{1}{R} \frac{\cosh \sqrt{\frac{27}{8}} \frac{y}{R}}{\sinh \sqrt{\frac{27}{8}} \frac{h}{R}}\right) \theta_{h} \omega^{2} \sin \omega t
\end{gathered}
$$

The pressure on the wall is

$$
p_{w}=-\rho \frac{R^{3}}{3} \frac{\partial \ddot{\theta}}{\partial y}\left(1-\frac{\cos ^{2} \phi}{3}-\frac{\sin ^{2} \phi}{4}\right) \cos \phi
$$

The resultant horizontal force exerted on the wall is

$$
\begin{aligned}
P & =-\pi \frac{11}{48} \rho \omega^{2} R^{4} \theta_{h} \sin \omega t \\
& =\frac{12}{11} M_{1} g \theta_{h} \sin \omega t
\end{aligned}
$$


This force is the same as that produced by an equivalent mass $M_{1}$ oscillating in a horizontal plane with motion

$$
\begin{aligned}
& x_{1}=A_{1} \sin \omega t \\
& M_{1}=M \frac{1}{4}\left(\frac{11}{12}\right)^{2} \sqrt{\frac{27}{8}} \frac{R}{h} \tanh \sqrt{\frac{27}{8}} \frac{h}{R} \\
& A_{1}=\theta_{h} \frac{\frac{12}{11} h}{\sqrt{\frac{27}{8} \frac{h}{R} \tanh \sqrt{\frac{27}{8} \frac{h}{R}}}}
\end{aligned}
$$

In order that $M_{1}$ exert the same moment as the fluid pressure on the wall it should be at an elevation above the bottom of

$$
h_{1}=h\left(1-\frac{1}{\sqrt{\frac{27}{8} \frac{h}{R} \tanh \sqrt{\frac{27}{8}} \frac{h}{R}}}\right)
$$

The pressure exerted on the bottom of the tank is

$$
p_{b}=-\rho \omega^{2} \sqrt{\frac{3}{8}} \frac{R^{2}}{\sinh \sqrt{\frac{27}{8}} \frac{h}{R}}\left(\frac{x}{R}-\frac{1}{3}\left(\frac{x}{R}\right)^{3}-\frac{1}{4} \frac{x}{R}\left(\frac{z}{R}\right)^{2}\right) \theta_{h} \sin \omega t
$$

This exerts a moment about the $\mathrm{z}$ axis equal to

$$
\frac{5}{32} \sqrt{\frac{3}{8}} \frac{\pi R^{5} \rho \omega^{2}}{\sinh \sqrt{\frac{27}{8}} \frac{h}{R}}
$$

Including this, the correct total moment on the tank is produced when

$$
h_{1}=h\left(1-\frac{\cosh \sqrt{\frac{27}{8} \frac{h}{R}-\frac{135}{88}}}{\sqrt{\frac{27}{8}} \frac{h}{R} \sinh \sqrt{\frac{27}{8}} \frac{h}{R}}\right)
$$

Elimiptical Tank

Proceeding in the same way as for the cylindrical tank, the impulsive pressure on the wall is given by equation (14)

$$
p_{w}=\rho \dot{u_{0}} h\left(y / h-\frac{1}{2}(y / h)^{2}\right) \sqrt{3} \tanh \sqrt{3} \frac{l}{h}
$$

with a similar expression for acceleration in the direction of the $y$ axis. 
For oscillations of the fluid, equations (21) apply and for the first mode about the minor axis

$$
\omega^{2}=\frac{g}{a} \sqrt{\frac{54}{15+\left(\frac{b}{a}\right)^{2}}} \tanh \sqrt{\frac{54}{15+\left(\frac{b}{a}\right)^{2}}} \frac{h}{a}
$$

where $2 a$ is the major axis of the ellipse and $2 b$ is the minor axis. For $h / a$ small this reduces to

$$
\omega=\frac{\sqrt{g h}}{a} \sqrt{\frac{54}{15+\left(\frac{b}{a}\right)^{2}}}
$$

Comparing this with the exact solution, Jeffreys (1924), it is found that $\omega$ is slightly overestimated with a maximum error less than 1 per cent.

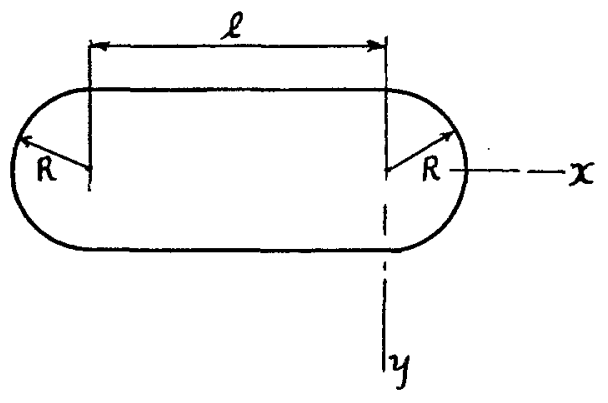

Fig. 7.

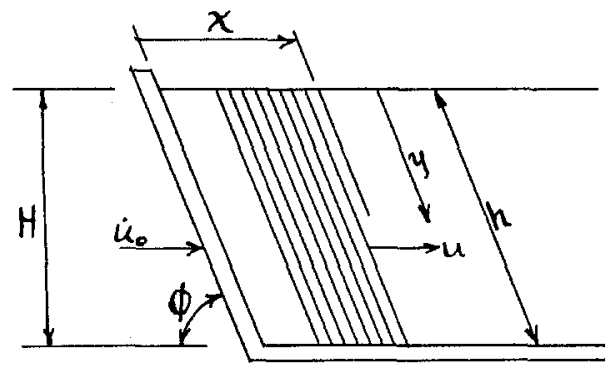

Fig. 8.

\section{COMPOSITE TANKS}

Symmetrical tanks formed of composite shapes such as that shown in figure 7 will have impulsive pressures given by equation (14) and oscillations described by equations (21). The tank shown in figure 7 has

$$
\begin{gathered}
I_{y}=\frac{2 R l^{3}}{12}+\pi R^{4}\left(\frac{1}{4}+\left(\frac{l}{R}+\frac{4}{3 \pi}\right)^{2}\right) \\
K_{y}=R l^{5}\left\{0.233\left(\frac{R}{l}\right)^{5}+0.627\left(\frac{R}{l}\right)^{4}+1.377\left(\frac{R}{l}\right)^{3}+0.197\left(\frac{R}{l}\right)^{2}\right. \\
\left.+0.131 \frac{R}{l}+0.0166\right\}
\end{gathered}
$$

\section{Rectangular Dam}

For a dam with sloping rectangular face and constraints on the flow as shown in figure 8 , the impulsive pressures are given by the following equations: 


$$
\begin{aligned}
v & =(h-y) \frac{d u}{d x}+u \cos \phi \\
\dot{u} & =\dot{u}_{0} \exp (-\sqrt{3} x / h) \\
\frac{\partial p}{\partial y} & =-\rho \dot{v} \\
p_{w} & =\rho \dot{u_{0}} h\left\{\left(\frac{y}{h}-\frac{1}{2}\left(\frac{y}{h}\right)^{2}\right) \sqrt{3}-\frac{y}{h} \cos \phi\right\} \\
P_{w} & =\rho \dot{u}_{0} h^{2}\left\{\frac{1}{\sqrt{3}}-\frac{\cos \phi}{2}\right\}
\end{aligned}
$$

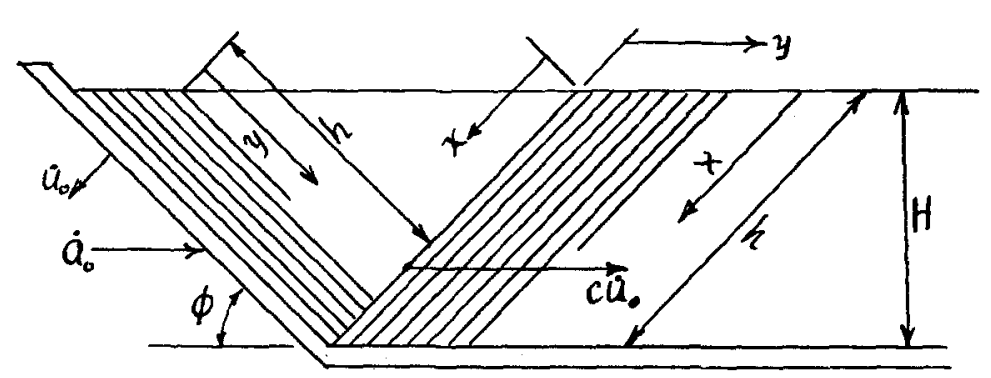

Fig. 9.

The resultant horizontal force on the dam is

$$
F_{h}=\rho \dot{u}_{0} H^{2} \frac{1}{\sin \phi}\left\{\frac{1}{\sqrt{3}}-\frac{\cos \phi}{2}\right\}
$$

For $90 \geqq \phi \geqq 55^{\circ}$, equation (48) overestimates $F_{h}$ by 6.5 per cent; for $\phi<55^{\circ}$ the accuracy of the preceding equation decreases and a different approximation must be used, as given below.

When $\phi<55^{\circ}$ the fluid may be divided into two regions as shown in figure 9 , where a rigid membrane lies along the $\mathrm{x}$ axis and has a horizontal acceleration $c \dot{u}_{0}$ such that the pressure force on each side of the membrane is the same. In the region to the left of the $\mathrm{x}$ axis the following equations describe the flow:

$$
\begin{aligned}
v & =(h-y) \frac{\partial u}{\partial x}+c u_{0} \cos \phi \\
\frac{\partial p}{\partial y} & =-\rho \dot{v}
\end{aligned}
$$


Applying Hamilton's Principle to the total kinetic energy in this region leads to the equation

$$
x^{2} \frac{d^{2} \dot{u}}{d x^{2}}+3 x \frac{d y}{d x}-3 \tan ^{2} \phi \dot{u}=3 c \dot{u}_{0} \cos \phi \tan \phi
$$

The appropriate solution is

$$
\begin{aligned}
& \dot{u}=\dot{u}_{0}\left\{\left(1-c \frac{\cos \phi}{\tan \phi}\right)\left(\frac{x}{l}\right)^{\alpha}+c \frac{\cos \phi}{\tan \phi}\right\} \\
& \alpha=\sqrt{1+3 \tan ^{2} \phi}-1
\end{aligned}
$$

The pressure in the fluid is

$$
p=-\rho \dot{u}_{0}\left\{\left(h y-\frac{1}{2} y^{2}\right) \frac{\alpha}{l}\left(1-c \frac{\cos \phi}{\tan \phi}\right)\left(\frac{x}{l}\right)^{\alpha-1}+c y \cos \phi\right\}
$$

In the region to the right of the $\mathrm{x}$ axis, figure 9 ,

$$
\begin{aligned}
& v=(l-x) \frac{d u}{d y}+u \cos \phi \\
& p=-\rho\left(l x-\frac{1}{2} x^{2}\right) \frac{d \dot{i}}{d y}+x \dot{u} \cos \phi \\
& P=\rho\left(\frac{l^{3}}{3} \frac{d \dot{u}}{d y}+\frac{l^{2}}{2} \dot{u} \cos \phi\right) \\
& \dot{u}=\dot{u}_{0} \exp \left(-\sqrt{3} \frac{y}{h}\right)
\end{aligned}
$$

Equating the pressure forces on the two sides of the membrane lying along the $x$ axis determines the value of the constant

$$
\begin{aligned}
& c=-\frac{1}{2 \beta \tan ^{2} \phi+\cos \phi-\frac{\cos \phi}{\tan \phi} \frac{\alpha}{\alpha+2}} \cdot \frac{\alpha}{\alpha+2} \\
& \beta=\left(\frac{1}{\sqrt{3}}-\frac{\cos \phi}{2}\right)
\end{aligned}
$$

The pressure on the inclined wall of the dam is thus

$$
p_{w}=-\rho \dot{a}_{0} H\left\{\left(\frac{y}{h}-\frac{1}{2}\left(\frac{y}{h}\right)^{2}\right) \frac{\alpha}{\tan \phi}\left(1-c \frac{\cos ^{2} \phi}{\sin \phi}\right)+c \frac{y}{h} \cos \phi\right.
$$


where $\dot{a}_{0}$ is the horizontal acceleration of the inclined face. The resultant horizontal force exerted against the inclined face is

$$
F_{h}=-\rho \dot{a_{0}} H^{2}\left\{\frac{\alpha}{\tan \phi}\left(1-c \frac{\cos \phi}{\tan \phi}\right)+\frac{c}{2} \cos \phi\right\}
$$

Equation (54) overestimates $F_{h}$ with a maximum error of 6 per cent at $\phi=25^{\circ}$.

\section{Flexible Retaining Wall}

An approximate analysis may be made of the effect of wall flexibility on water pressures. Suppose the fluid is retained by a vertical cantilever wall which is stiff to the degree that wave propagation in the wall may be neglected. As shown in figure 10 , let the fluid be restrained by membranes whose shapes are proportional to the deflected shape of the wall. For a sinusoidal vibration the horizontal velocity of a fluid particle is $u f(y) \sin \omega t$, and the vertical velocity is

$$
v=\frac{\partial u}{\partial x} \int_{y}^{h} f(y) d y \sin \omega t
$$

Applying Hamilton's Principle, there is obtained

$$
\begin{aligned}
& \frac{d^{2} \dot{u}}{d x^{2}}+\frac{A}{B} \dot{u}=0 \\
& \dot{u}=\dot{u}_{0} \exp \left(-\sqrt{\frac{A}{B}} x\right) \\
& A=\int_{0}^{h}(f(y))^{2} d y \\
& \left.B=\int_{0}^{h}\left(\int_{y}^{h} f(y) d y\right)\right)^{2} d y
\end{aligned}
$$

The pressure on the wall is

$$
p_{w}=\rho \dot{u}_{0} \omega^{2} \sqrt{\frac{A}{B}} \int_{0}^{y} \int_{y}^{h} f(y) d y d y \sin \omega t
$$

and the resultant force on the wall is

$$
P=\rho \dot{u}_{0} \omega^{2} \sqrt{\frac{A}{B}} \int_{0}^{h} \int_{0}^{y} \int_{y}^{h} f(y) d y d y d y \sin \omega t
$$

For a wall of uniform cross section, if we approximate the actual pressure by 
$p_{0} \sin (\pi / 2)(y / h)$ and compute the deflected shape of the wall, there is obtained

$$
\begin{gathered}
f(y)=\dot{u}_{0}\left(1-\beta+\beta \sin \frac{\pi}{2} \frac{x}{h}\right) \\
\beta=\frac{P}{\dot{u}_{0}} \frac{h^{3}}{\left(\frac{\pi}{4}\right)^{4} E I}
\end{gathered}
$$

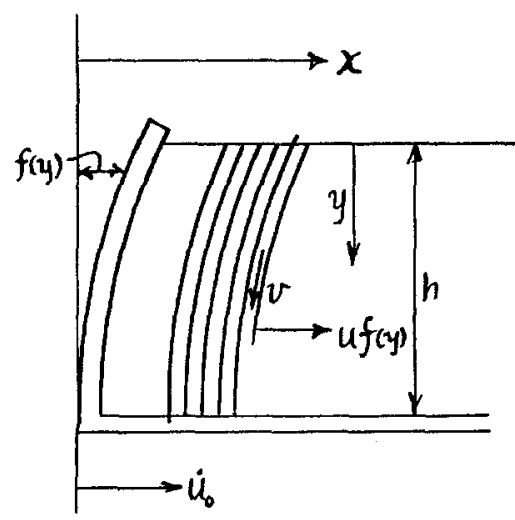

Fig. 10.

where $P$ is the total force exerted on the wall. With this $f(y)$ the pressure and force are computed to be

$$
\begin{gathered}
p_{w}=\rho h \dot{u}_{0} \omega^{2} \sqrt{3} \sqrt{\frac{1-1.68 \beta+1.18 \beta^{2}}{1+2.44 \beta+1.63 \beta^{2}}}\left((1-\beta)\left(\frac{y}{h}-\frac{1}{2}\left(\frac{y}{h}\right)^{2}\right)+\left(\frac{2}{\pi}\right)^{2} \beta \sin \frac{\pi}{2} \frac{y}{h}\right) \\
P=\frac{\rho h^{2} \dot{u}_{0} \omega^{2}}{\sqrt{3}} \sqrt{\frac{1-1.68 \beta+1.18 \beta^{2}}{1+2.4 \beta+1.63 \beta^{2}}}(1-0.22 \beta)
\end{gathered}
$$

The latter equation may be written

$$
\begin{aligned}
\sqrt{3} N & =\sqrt{\frac{1-1.68 \beta}{1+2.44 \beta}+1.18 \beta^{2}} \frac{\left(1-03 \beta^{2}\right.}{\beta} \\
N & =\left(\frac{\pi}{2}\right)^{4} \frac{E I}{\rho w^{2} h^{5}}
\end{aligned}
$$

For a given $\omega$, that is, a given $N$, this equation gives the appropriate value of $\beta$. Figure 11 gives a graph of $N$ vs $\beta$ and also shows how the total force on the wall is reduced by wall flexibility. For a rigid wall, $E I=\infty$, the preceding equation overestimates $P$ by 6 per cent. 


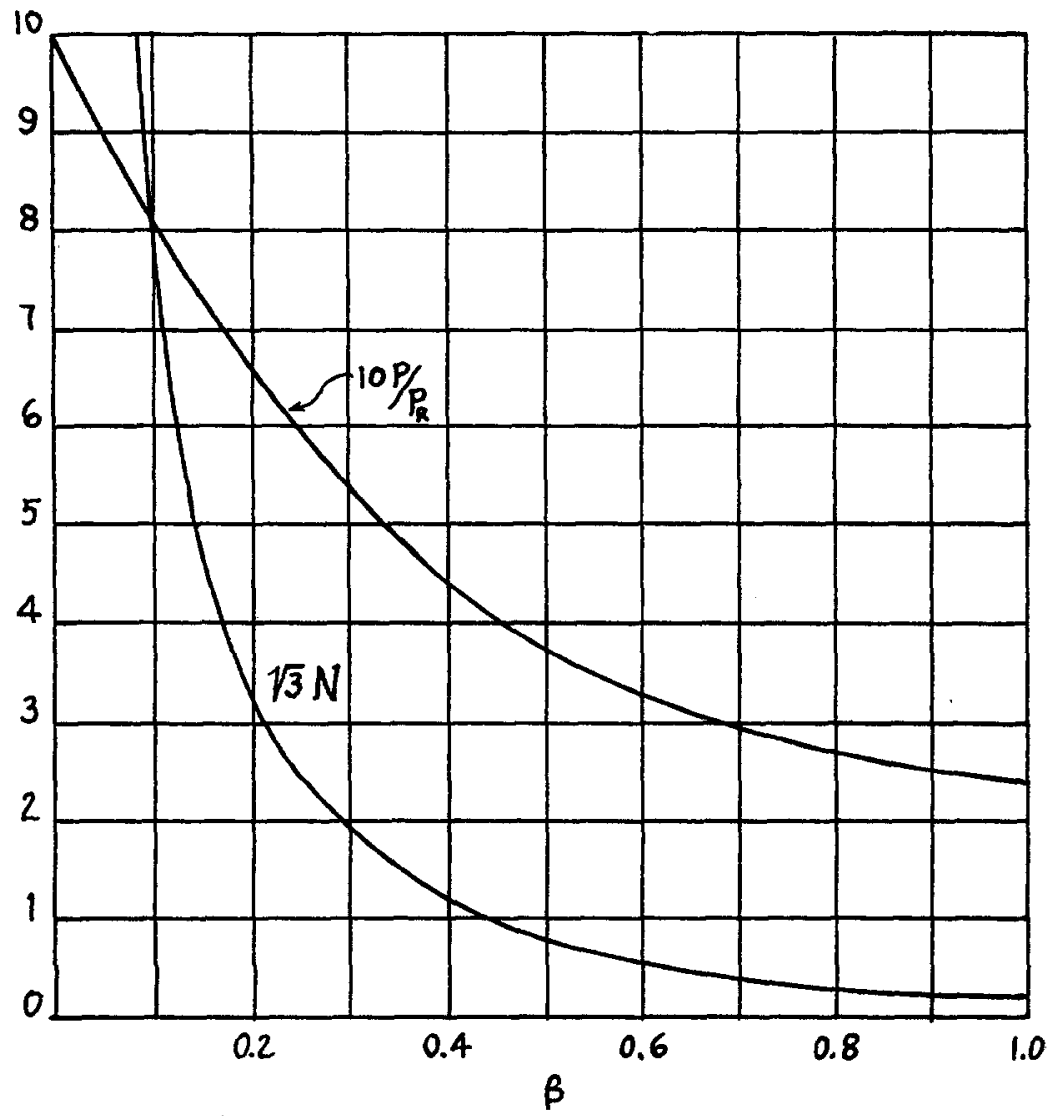

Fig. 11.

\section{Numerical Example}

Consider a cylindrical tank of $40 \mathrm{ft}$. radius and $25 \mathrm{ft}$. depth of water. Suppose the tank to be given a horizontal acceleration in the x direction of $\dot{u}_{0}=0.1 \mathrm{~g}$ and let this acceleration persist for $1 / 4$ second and during the following $1 / 4$ second let $\dot{u}_{0}=$ $-0.1 g$, so that the tank comes to rest with a total displacement of $0.2 \mathrm{ft}$. During this time the impulsive pressure on the wall of the tank is given by equation (31), which for the present problem takes the form

$$
\mathbf{p}_{\mathrm{w}} \sqrt{3}(62.4)(0.1)(25)\left(\frac{y}{h}-\frac{1}{2}\left(\frac{y}{h}\right)^{2}\right) \tanh \left(\sqrt{3} \frac{40}{25} \cos \phi\right)
$$

From equation (34) the total impulsive force exerted on the tank is

$$
P=0.1 \mathrm{~W} \frac{\tanh \sqrt{3} \frac{40}{25}}{\sqrt{3} \frac{40}{25}}=0.36 \mathrm{~W}=280,000 \mathrm{lbs} .
$$


The oscillations set up in the fluid are determined from equations (37), etc.

$$
\begin{aligned}
& \omega^{2}=\frac{g}{40} \sqrt{\frac{27}{8}} \tanh \sqrt{\frac{27}{8}} \frac{25}{40}=2.6 \\
& \frac{2 \pi}{\omega}=3.9 \text { seconds period of vibration }
\end{aligned}
$$

The force exerted by the fluid during the oscillations is the same as that exerted by a simple oscillator (figure 5), which has

$$
M_{1}=M \frac{1}{4}\left(\frac{11}{12}\right)^{2} \sqrt{\frac{27}{8}} \frac{40}{25} \tanh \sqrt{\frac{27}{8}} \frac{25}{40}=0.50 M
$$

Since the natural period ( $3.9 \mathrm{sec}$.) is long as compared with the $1 / 2 \mathrm{sec}$. duration of the ground acceleration, the net effect of the $0.1 \mathrm{~g}$ acceleration is to generate an initial displacement of $0.2 \mathrm{ft}$. of $M_{1}$ relative to the tank wall and the consequent motion of $M_{1}$ is

$$
x_{1}=0.2 \sin \omega t
$$

The maximum $x_{1}$ is $0.2 \mathrm{ft}$, and hence from equation (41) the maximum $\theta_{h}$ is

$$
\theta_{h}=0.065 g \frac{11}{12} \sqrt{\frac{27}{8}} \frac{1}{40} \tanh \sqrt{\frac{27}{8}} \frac{25}{40}=0.008 \text { radians }
$$

The pressure on the wall at this $\theta_{h}$ is given by equations (37) and (39). The total force exerted on the wall is given by equation (40).

$$
P=\frac{12}{11}(0.5 M g)(0.008) \sin \omega t=33,000 \sin \omega t
$$

The amplitude of the water surface oscillations can be determined from equations (30) and (39). The fluid pressure against the wall at $y=h$ and $\phi=0$ is found from equation (39) to be $17 \mathrm{lbs}$. per sq. ft., and from equation (30) the amplitude of the water surface is

$$
d=\frac{17}{\rho\left(g-x \ddot{\theta}_{h}\right)}=0.28 \mathrm{ft}
$$

In summary, during the first $1 / 4$ second the fluid exerts a force of $280,000 \mathrm{lbs}$. against the tank wall, in the negative $x$ direction; during the next $1 / 4$ second the $280,000 \mathrm{lbs}$. force is in the $+\mathrm{x}$ direction; following this the only force against the wall is the oscillating force the amplitude of which is $30,000 \mathrm{lbs}$. and the period 3.9 sec. During this oscillation the maximum amplitude of the water surface is $0.28 \mathrm{ft}$. above the horizontal position. 


\section{REFERENCES}

H. Jeffreys (1924), "Free Oscillations of Water in an Elliptical Lake," Proc. London Math. Soc., Vol. 23, 1924 .

H. Lamb (1932), Hydrodynamics (Cambridge Univ. Press, 1932).

H. M. Westergaard (1933), "Water Pressures on Dams during Earthquakes," Trans. Am. Soc. Civ. Eng., Vol. 98, 1933.

L. M. Hoskins and L. S. Jacobsen (1934), "Water Pressure in a Tank Caused by a Simulated Earthquake, Bull. Seism. Soc. Am., Vol. 24, 1934.

L. S. Jacobsen (1949), "Impulsive Hydrodynamics of Fluid Inside a Cylindrical Tank and of a Fluid Surrounding a Cylindrical Pier," Bull. Seism. Soc. Am., Vol. 39, 1949.

P. W. Werner and K. J. Sundquist (1949), "On Hydrodynamic Earthquake Effects," Trans. Am. Geophys. Union, Vol. 30, 1949.

L. S. Jacobsen and R. S. Ayre (1951), "Hydrodynamic Experiments with Rigid Cylindrical Tanks Subjected to Transient Motions," Bull. Seism. Soc. Am., Vol. 41, 1951.

E. W. Graham and A. M. Rodriguez (1952), "Characteristics of Fuel Motion Which Affect Airplane Dynamics," Jour. Applied Mechanics, Vol. 19, No. 3, 1952.

C. N. Zangar (1953), "Hydrodynamic Pressures on Dams Due to Horizontal Earthquakes," Proc. Soc. Exper. Stress Analysis, Vol. 10, No. 2, 1953.

G. W. Housner (1954), Earthquake Pressures on Fluid Containers (California Institute of Technology, 1954).

DIVISION OF ENGINEERING,

California Institute of Technology,

Pasadena, CAlif. 